\title{
HOW LARGE DELAYS BUILD UP IN A GI/G/1 QUEUE *
}

\author{
V. ANANTHARAM
}

School of Electrical Engineering, Phillips Hall, Cornell University, Ithaca, NY 14853, U.S.A.

Received 1 April 1988; revised 12 June 1989

\begin{abstract}
Let $W_{k}$ denote the waiting time of customer $k, k \geqslant 0$, in an initially empty GI/G $/ 1$ queue. Fix $a>0$. We prove weak limit theorems describing the behaviour of $W_{k} / n, 0 \leqslant k \leqslant n$, given $W_{n}>n a$. Let $X$ have the distribution of the difference between the service and interarrival distributions. We consider queues for which Cramer type conditions hold for $X$, and queues for which $X$ has regularly varying positive tail.

The results can also be interpreted as conditional limit theorems, conditional on large maxima in the partial sums of random walks with negative drift.

Keywords: Weak limit theorems, Cramer type conditions, random walks with negative drift, waiting time.
\end{abstract}

\section{Introduction}

Consider an initially empty $\mathrm{GI} / \mathrm{G} / 1$ queue. Customer 0 arrives at time 0 and customer $k$ at time $A_{1}+\ldots+A_{k}$, where $A_{i}, i=1,2, \ldots$ are i.i.d.. Let $B_{i}, i=$ $1,2, \ldots$ be i.i.d. service times, $B_{k+1}$ being the service time of customer $k$. If we let $X_{k}=B_{k}-A_{k}$, then $X_{i}, i=1,2, \ldots$ are i.i.d.. Let $E X_{1}=-\mu, \mu>0$. The waiting time $W_{k}$ of customer $k$ before receiving service is given by the equations:

$$
\begin{aligned}
& W_{0}=0, \\
& W_{k+1}=\max \left(0, W_{k}+X_{k+1}\right), k>0 .
\end{aligned}
$$

Let $C$ denote $C[0,1]$, the space of continuous functions on the unit interval with the topology of uniform convergence, and $D$ denote $D[0,1]$, the space of right continuous functions with left limits on the unit interval, with the topology of uniform convergence. For each $n \geqslant 1$, let $w_{n}(\cdot) \in C$ denote the polygonal path, and $\hat{w}_{n}(\cdot) \in D$ denote the piecewise constant right continuous path, constructed from the points

$$
\left(\frac{k}{n}, \frac{W_{k}}{n}\right), 0 \leqslant k \leqslant n .
$$

* Research supported by the NSF under Grant NCR 8710840 and under the PYI Award NCR 8857731 . 
Fix $a>0$. Let $P_{n}$ denote the conditional distribution $\left(w_{n}(\cdot) \mid W_{n}>n a\right)$, and $\hat{P}_{n}$ the conditional distribution $\left(\hat{w}_{n}(\cdot) \mid W_{n}>n a\right) . P_{n}$ (resp. $\left.\hat{P}_{n}\right)$ is a probability distribution on $C$ (resp. $D$ ).

In section 2, thm. 2.1, we identify the weak limit of $\left\{P_{n}\right\}$ under the following moment conditions on $X_{1}$ :

(C) Let $m(t)=E \exp t X_{1}$. There is an interval $S=\left(t_{-}, t_{+}\right)$containing 0 , such that:

$$
\begin{aligned}
& m(t)<\infty \text { for all } t \in S, \\
& \frac{m^{\prime}(t)}{m(t)} \rightarrow \infty \text { as } t \rightarrow t_{+}, \\
& \frac{m^{\prime}(t)}{m(t)} \rightarrow-\infty \text { as } t \rightarrow t_{-} .
\end{aligned}
$$

This situation covers the $M / M / 1$ queue and a large class of GI/G/1 queues with rapidly decreasing tail distributions for $X_{1}$.

In section 3 , thm. 3.1, we identify the weak limit of $\left\{\hat{P}_{n}\right\}$ under the following moment conditions on $X_{1}$ :

(D) There is $q>0$ and a slowly varying function $L(\cdot)$ such that:

$$
\begin{aligned}
& E X_{1}^{2}<\infty, \\
& P\left(X_{1}>x\right)=x^{-q} L(x) .
\end{aligned}
$$

This situation covers a large class of GI/G/1 queues where the service time distribution has a fat tail.

\section{Cramer type conditions}

Throughout this section we assume the moment conditions (C). Then $m(\cdot)$ is an analytic, strictly convex function on $S$, as is $\log m(\cdot)$. Note that $m^{\prime}(0) / m(0)$ $=-\mu$. Let $t_{0}>0$ be the unique point where $m\left(t_{0}\right)=1$, and let $z_{0}$ denote $m^{\prime}\left(t_{0}\right) / m\left(t_{0}\right)$. See fig. 1 .

The main results of this section is the following:

\section{THEOREM 2.1}

Assume the moment conditions (C). If $a \leqslant z_{0}$, let $t(a)=1-\left(a / z_{0}\right)$ and $p_{a}(\cdot) \in C$ be defined by:

$$
\begin{aligned}
& p_{a}(t)=0, \quad 0 \leqslant t \leqslant t(a), \\
& p_{a}(t)=z_{0}(t-t(a)), \quad t(a) \leqslant t \leqslant 1 .
\end{aligned}
$$

If $a \geqslant z_{0}$, let $p_{a}(\cdot) \in C$ be defined by:

$$
p_{a}(t)=t a, \quad 0 \leqslant t \leqslant 1 \text {. }
$$

See fig. 2 . 


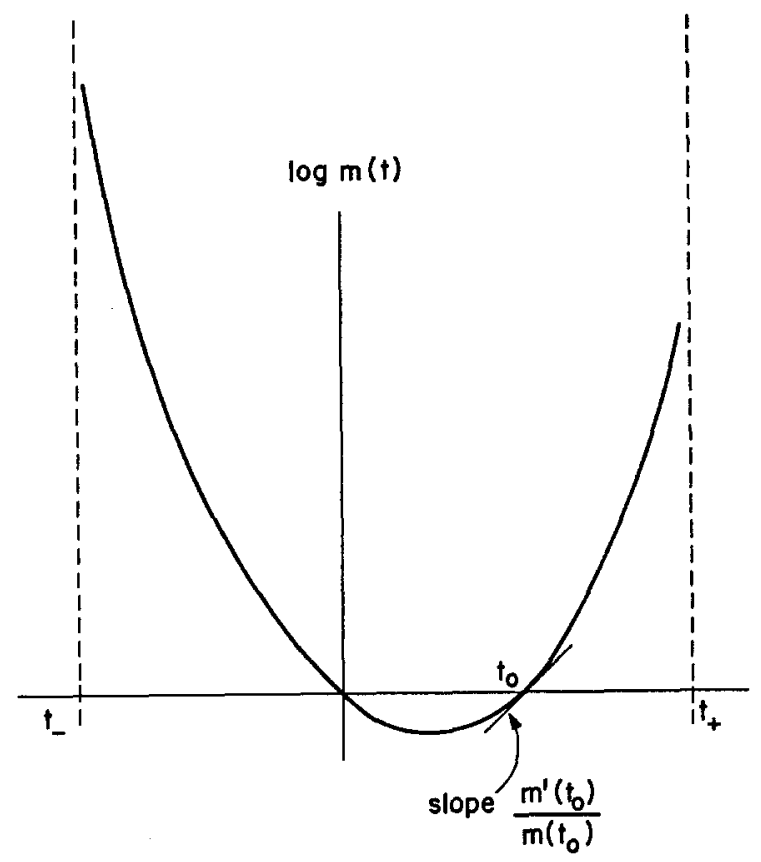

Fig. 1.

We have

$$
P_{n} \stackrel{w}{\rightarrow} \delta_{p_{a}}
$$

where $\delta_{p_{a}}$ denotes the probability distribution concentrated on $p_{a}(\cdot)$, and $\stackrel{w}{\rightarrow}$ denotes weak convergence of probabilities on $C$.
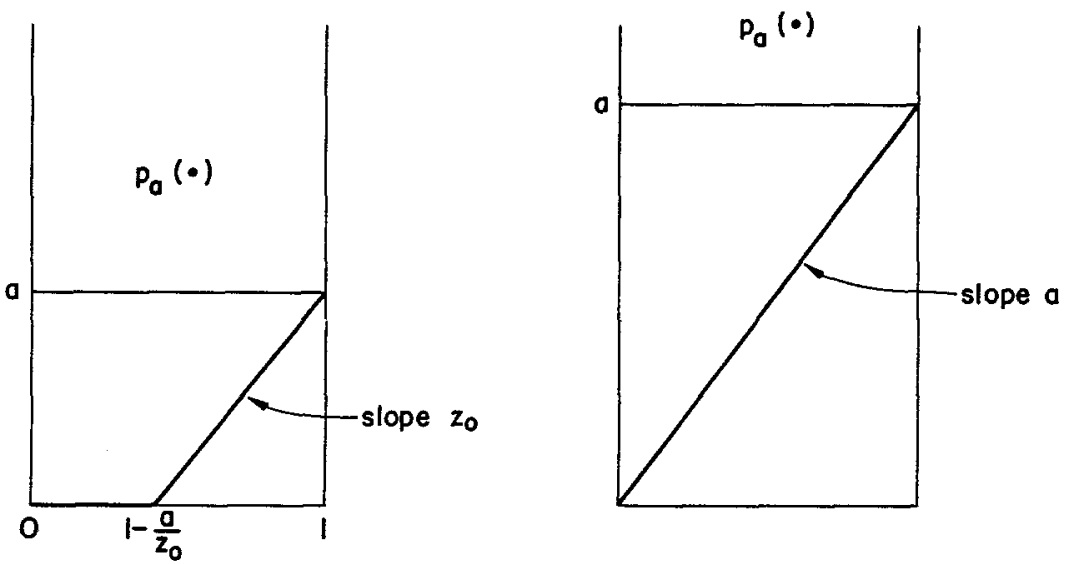

$$
a=z_{0}
$$$$
a=z_{0}
$$

Fig. 2. 
For the meaning of weak convergence, see Billingsley, [5]. Heuristically, thm. 2.1 states that the most likely way in which the waiting time of customer $n$ builds up to $n a$ as follows: If $a \leqslant z_{0}$, customers $k, k \leqslant[n t(a)]$, encounter essentially typical behaviour in the queue, after which the waiting time of customers builds up essentially linearly. If $a \geqslant z_{0}$, waiting time starts building up right away and builds up essentially linearly.

Let $Y_{1}, Y_{2}, \ldots$ be i.i.d. with the distribution of $X_{1}$ and let:

$$
\begin{aligned}
& S_{0}=0, \\
& S_{k}=Y_{1}+Y_{2}+\ldots+Y_{k} .
\end{aligned}
$$

From the points

$$
\left(\frac{k}{n}, \frac{S_{k}}{n}\right), 0 \leqslant k \leqslant n,
$$

we construct the piecewise linear, continuous path $s_{n}(\cdot) \in C$. Let

$$
M_{n}=\sup _{0 \leqslant k \leqslant n} S_{k}
$$

and let $Q_{n}$ denote the conditional distribution of $\left(s_{n}(\cdot) \mid M_{n}>n a\right)$. Let $\Psi: C \rightarrow C$ be given by

$$
\Psi(\phi)(t)=\left[\sup _{1-t \leqslant s \leqslant 1} \phi(s)\right]-\phi(1-t) .
$$

Note that $\Psi$ is continuous.

\section{LEMMA 2.2}

For each $n=1,2, \ldots$, we have

$$
P_{n}=\Psi_{*}\left(Q_{n}\right)
$$

where $\Psi_{*}\left(Q_{n}\right)$ denotes the distribution induced on $C$ by $\Psi$, i.e., for any Borel set $B \subseteq C, \Psi_{*}\left(Q_{n}\right)(B)=Q_{n}\left(\Psi^{-1}(B)\right)$.

\section{Proof}

From eqns. (1.1), we see that

$$
W_{k}=\max \left(0, X_{k}, X_{k}+X_{k-1}, \ldots, X_{k}+X_{k-1}+\ldots+X_{1}\right) .
$$

The claim follows easily.

Lemma 2.2 reduces the limit behaviour of $\left\{P_{n}\right\}$ to that of $\left\{Q_{n}\right\}$. In fact, by Billingsley, Thm. 5.1, [5], if we can show that $Q_{n} \stackrel{w}{\rightarrow} Q$ for some probability distribution $Q$ on $C$, then $P_{n} \stackrel{w}{\rightarrow} \Psi_{*}(Q)$. Thus thm. 2.1 is a direct consequence of the following: 
THEOREM 2.3

Assume the moment conditions (C). If $a \leqslant z_{0}$, let $\tau(a)=a / z_{0}$ and let $q_{a}(\cdot) \in C$ be defined by:

$$
\begin{array}{ll}
q_{a}(t)=t z_{0}, & 0 \leqslant t \leqslant \tau(a), \\
q_{a}(t)=a-\mu(t-\tau(a)), & \tau(a) \leqslant t \leqslant 1 .
\end{array}
$$

If $a \geqslant z_{0}$, let $q_{a}(\cdot) \in C$ be defined by:

$$
q_{a}(t)=t a, \quad 0 \leqslant t \leqslant 1 .
$$

See fig. 3.

We have

$$
Q_{n} \stackrel{w}{\rightarrow} \delta_{q_{a}} .
$$

We proceed to prove thm. 2.3. We first introduce the convex dual of $\log m(\cdot)$,

$$
I(z)=\sup _{t \in S} z t-\log m(t), z \in R
$$

$I(\cdot)$ is nonnegative, finite, and strictly convex. If $\bar{t}$ denotes the unique point at which $m^{\prime}(\bar{t}) / m(\bar{t})=z$, then

$$
I(z)=z \bar{t}-\log m(\bar{t}) .
$$

It is easily checked that $I(-\mu)=0$. It is also easy to see that $\log m(\cdot)$ is the convex dual of $I(\cdot)$, i.e.,

$$
\log m(t)=\sup _{z \in R} t z-I(z), \quad t \in S .
$$

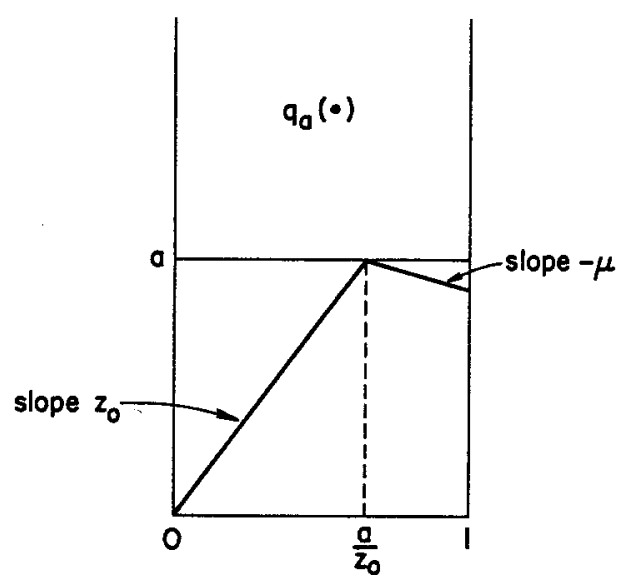

$0 \leq \mathbf{z}_{0}$

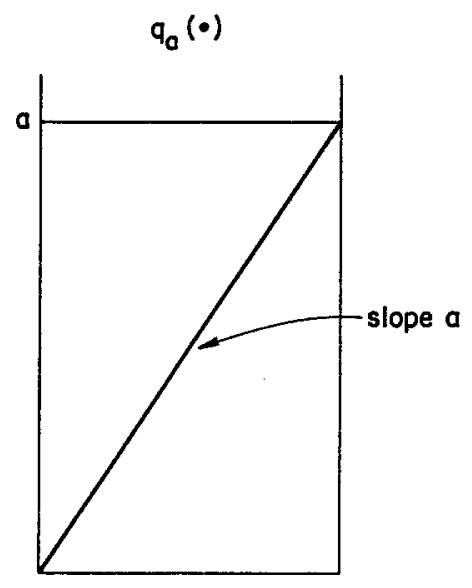

$a \geq z_{0}$

Fig. 3. 
The Skorohod metric on $C$ is defined by

$$
d_{S}(f, g)=\inf _{\lambda, \eta}\left\{\sup _{t \in[0,1]}|f(\lambda(t))-g(\eta(t))|+\sup _{t \in[0,1]}|\lambda(t)-\eta(t)|\right\}
$$

where $f(\cdot), g(\cdot) \in C$ and the infimum is taken over all continuous, strictly increasing functions $\lambda(t), \eta(t), 0 \leqslant t \leqslant 1$, such that $\lambda(0)=\eta(0)=0$ and $\lambda(1)=$ $\eta(1)=1$. In future we will refer to such functions as "time changes". It is well known that the Skorohod metric induces the topology of uniform convergence on $C$, see, e.g., Bergstrom, thm. 3, pg. 169, [4]. Let $E$ denote the completion of $C$ in the Skorohod metric. We also recall the standard notation

$$
\|f\|_{\infty}=\max _{t \in[0,1]}|f(t)|, \quad f \in C .
$$

Let $\Gamma \subseteq C$ denote the set of all continuous, piecewise linear functions with finitely many break points, and starting at 0 . If $f(\cdot) \in \Gamma$, with slope $f_{i}^{\prime}$ on the interval $\left(t_{i-1}, t_{i}\right), 1 \leqslant i \leqslant N$, where $0=t_{0}<t_{1}<t_{2}<\ldots<t_{N}=1$, let

$$
h(f)=\sum_{i=1}^{N}\left(t_{i}-t_{i-1}\right) I\left(f_{i}^{\prime}\right) .
$$

Extend the functional $h$ to $E$ by setting

$$
h(f)=\inf \liminf _{n \rightarrow \infty} h\left(f_{n}\right), \quad f \in E,
$$

where the infimum is taken over all sequences $\left\{f_{n}\right\} \subseteq \Gamma$ such that $d_{S}\left(f_{n}, f\right) \rightarrow 0$ as $n \rightarrow \infty$. Appealing to the convexity of $I(\cdot)$, it can be easily shown that this extension does not change the definition of $h$ on $\Gamma$. For details, see [9]. It can also be shown that for $f(\cdot) \in C, h(f)=\infty$ unless $f(0)=0$ and $f(\cdot)$ has finite total variation.

For any subset $G \subseteq E$ let

$$
H(G)=\inf _{f \in G} h(f) .
$$

Our main tool in the proof of thm. 2.3 is the following special case of theorem 2 of Mogulskii, [9]:

\section{THEOREM 2.4}

Assume the moment conditions (C). For an arbitrary Borel subset $G \subseteq C$, let $G^{0}$ denote its interior and $\bar{G}^{E}$ its closure in $E$. If $H\left(G^{0}\right)=H\left(\bar{G}^{E}\right)$ then

$$
\log P\left(s_{n}(\cdot) \in G\right)=-n H(G)+o(n) .
$$

The following sequence of lemmas will be used in the proof of thm. 2.3. Let

$$
X=\left\{f \in C \mid \sup _{t \in[0,1]} f(t)>a\right\} .
$$

Note that $X$ is open, and $Q_{n}(X)=1$ for all $n$. 
LEMMAS 2.5

$H(X)=h\left(q_{a}\right)$.

Proof

First we show that $H(X) \geqslant h\left(q_{a}\right)$. Let $f \in X \cap \Gamma$. We may assume that $f(0)=0$, else $h(f)=\infty$. Let $\bar{t}=\inf \{t \in[0,1] \mid f(t)>a\}$. Then $f(\bar{t})=a$. Denote by $r_{t}$ the piecewise linear path connecting $(0,0)$ to $(t, a)$ and then dropping off with slope $-\mu$. By convexity of $I(\cdot)$ and the fact that $I(-\mu)=0$, we have

$$
h(f) \geqslant h\left(r_{\bar{t}}\right)=\bar{t} I\left(\frac{a}{\bar{t}}\right) .
$$

Note that $z_{0}$ satisfies

$$
I^{\prime}\left(z_{0}\right)=\frac{I\left(z_{0}\right)}{z_{0}} .
$$

Further, $I(z) / z$ decreases with $z$ for $z \in\left(0, z_{0}\right)$ and increases with $z$ for $z \in\left(z_{0}, \infty\right)$, see fig. 4 . From this, it follows easily that

$$
\inf _{t \in[0,1]} t I\left(\frac{a}{t}\right)=I(a) \text { if } a>z_{0},
$$

and

$$
\inf _{t \in[0,1]} t I\left(\frac{a}{t}\right)=\frac{a}{z_{0}} I\left(z_{0}\right) \text { if } a \leqslant z_{0},
$$

i.e.,

$$
\inf _{t \in[0,1]} h\left(r_{t}\right)=h\left(q_{a}\right) .
$$

Hence, $h(f) \geqslant h\left(q_{a}\right)$ for all $f \in X \cap \Gamma$.

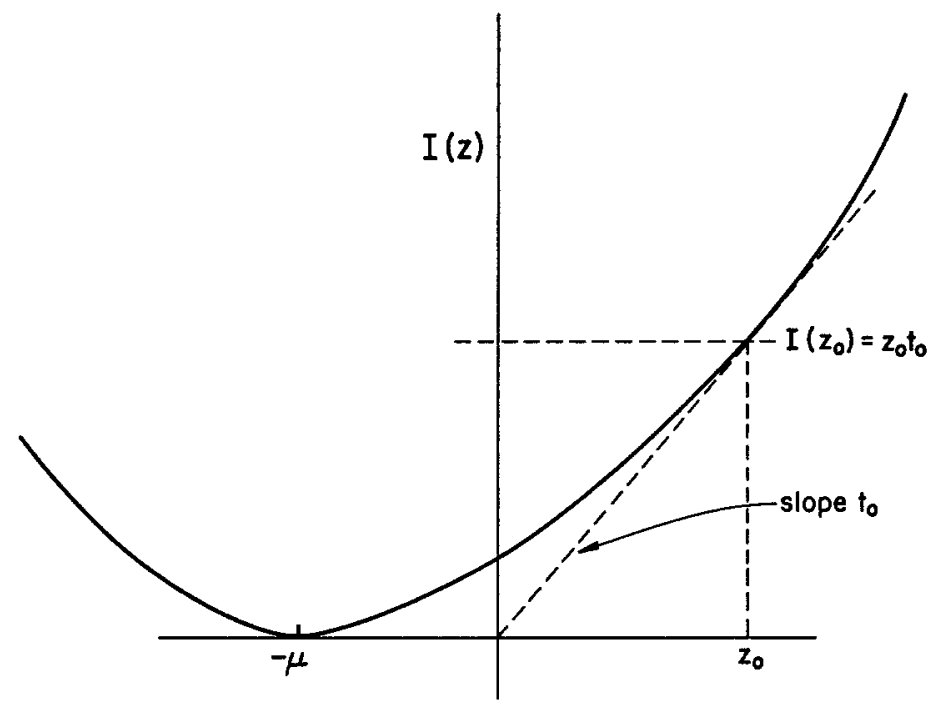

Fig. 4. 
Now, pick arbitrary $g \in X$ and $\left\{f_{n}\right\} \subseteq \Gamma$ such that $d_{S}\left(f_{n}, g\right) \rightarrow 0$ as $n \rightarrow \infty$. Since the Skorohod metric induces the topology of uniform convergence on $C$ we have $\left\|f_{n}-g\right\|_{\infty} \rightarrow 0$ as $n \rightarrow \infty$. Since $X$ is open, we see that $\left\{f_{n}\right\}$ eventually lies in $X \cap \Gamma$. So, liminf $h\left(f_{n}\right) \geqslant h\left(q_{a}\right)$. Varying $\left\{f_{n}\right\}$, we have $h(g) \geqslant h\left(q_{a}\right)$, by Defn. (2.1). Since $g \in X$ was arbitrary we have $H(X) \geqslant h\left(q_{a}\right)$, by defn. (2.2).

Finally, the sequence

$$
q_{a+1 / n}(\cdot) \in X \cap \Gamma
$$

converges uniformly to $q_{a}(\cdot)$. Hence $H(X) \leqslant h\left(q_{a}\right)$, completing the proof.

\section{LEMMA 2.6}

$$
H(X)=H\left(\bar{X}^{E}\right)
$$

\section{Proof}

Let $g \in \bar{X}^{E}$ and $\left\{f_{n}\right\} \subseteq \Gamma$ such that $d_{S}\left(f_{n}, g\right) \rightarrow 0$ as $n \rightarrow \infty$. We claim that $\liminf _{n \rightarrow \infty}\left\|f_{n}\right\|_{\infty} \geqslant a$. Since $g \in \bar{X}^{E}$, there is $\left\{g_{n}\right\} \subseteq X \cap \Gamma$ such that $d_{S}\left(g_{n}, g\right)$ $\rightarrow 0$ as $n \rightarrow \infty$. Suppose $\liminf _{n \rightarrow \infty}\left\|f_{n}\right\|_{\infty}<a-\epsilon, \epsilon>0$. We can find $N(\epsilon)$ so large such that $d_{S}\left(g_{m}, f_{n}\right)<\epsilon / 2$ for all $m, n \geqslant N(\epsilon)$. Let $N>N(\epsilon)$ be such that $\left\|f_{N}\right\|_{\infty}<a-\epsilon$. Choose $m>N(\epsilon)$ and let $\bar{t} \in[0,1]$ be such that $g_{m}(\bar{t})>a$. For any time changes $\lambda(\cdot)$ and $\eta(\cdot)$, we have,

$$
\sup _{t \in[0,1]}\left|g_{m}(\lambda(t))-f_{N}(\eta(t))\right| \geqslant\left|g_{m}(\bar{t})-f_{n}\left(\eta\left(\lambda^{-1}(\bar{t})\right)\right)\right|>\epsilon / 2 .
$$

This contradiction establishes the claim.

Since $\liminf _{n \rightarrow \infty}\left\|f_{n}\right\|_{\infty} \geqslant a$, given $\epsilon>0$, we can find $N(\epsilon)$ so large that $\left\|f_{n}\right\|_{\infty}>a-\epsilon$ for all $n \geqslant N(\epsilon)$. By lemma 2.5 it follows that $\liminf _{n \rightarrow \infty} f_{n} \geqslant$ $h\left(q_{a-\epsilon}\right)$. Since this applies to every $\left\{f_{n}\right\} \subseteq \Gamma$ converging to $g$, we have $h(g) \geqslant$ $h\left(q_{a-\epsilon}\right)$. Since $g \in \bar{X}^{E}$ was arbitrary, we have $H\left(\bar{X}^{E}\right) \geqslant h\left(q_{a-\epsilon}\right)$. Letting $\epsilon \rightarrow 0$ establishes the lemma.

Let

$$
\bar{B}\left(q_{a}, \epsilon\right)=\left\{f \in C\left|\sup _{t \in[0,1]}\right| f(t)-q_{a}(t) \mid \leqslant \epsilon\right\},
$$

and

$$
B\left(q_{a}, \epsilon\right)=\left\{f \in C\left|\sup _{t \in[0,1]}\right| f(t)-q_{a}(t) \mid<\epsilon\right\} .
$$

Let $Y_{\epsilon}=X-\bar{B}\left(q_{a}, \epsilon\right)$. Then $Y_{\epsilon}$ is open in $C$. We also have:

\section{LEMMA 2.7}

Given $\epsilon>0$, there is $\eta>0$ such that $H\left(Y_{\epsilon}\right) \geqslant h\left(q_{a}\right)+\eta$. 
Proof

It suffices to find $\eta>0$ such that $h(f)>h\left(q_{a}\right)+\eta$ for all $f \in Y_{\epsilon} \cap \Gamma$. For, if $g \in Y_{\epsilon}$ and $\left\{f_{n}\right\} \subseteq \Gamma$ is such that $d_{S}\left(f_{n}, g\right) \rightarrow 0$ as $n \rightarrow \infty$, then, since $Y_{\epsilon}$ is open, $\left\{f_{n}\right\}$ eventually consists of elements of $Y_{\epsilon} \cap \Gamma$, from which it follows that $h(g) \geqslant h\left(q_{a}\right)+\eta$.

Pick $0<\delta<\min \left(\epsilon / 3 z_{0}, \epsilon / 3 \mu\right)$. Using the notation in the proof of lemma 2.5, we have

$$
\left\|r_{t}-q_{a}\right\|_{\infty}<\epsilon / 3 \text { for all } t \in(\tau(a)-\delta, \tau(a)+\delta) \cap[0,1] .
$$

We also observe that there is $\eta_{1}>0$ such that

$$
h\left(r_{1}\right)>h\left(q_{a}\right)+\eta_{1} \text { for all } t \notin(\tau(a)-\delta, \tau(a)-\delta, \tau(a)+\delta), 0 \leqslant t \leqslant 1 .
$$

Given $f \in Y_{\epsilon} \cap \Gamma$, let $\bar{t}=\inf \{t \in[0,1] \mid f(t)>a\}$. If $\bar{t} \notin(\tau(a)-\delta, \tau(a)+\delta)$, we directly observe that $h(f) \geqslant h\left(r_{t}\right)$. If $\bar{t} \in(\tau(a)-\delta, \tau(a)+\delta)$, we note that $\left\|f-r_{t}\right\|_{\infty}>2 \epsilon / 3$. Pick $t^{*} \in[0,1]$ such that $\left|f\left(t^{*}\right)-r_{t}\left(t^{*}\right)\right|>2 \epsilon / 3$.

We distinguish 4 cases:

Case 1: $f\left(t^{*}\right)>r_{i}\left(t^{*}\right)+2 \epsilon / 3$ and $t^{*}<\bar{t}$.

Let $p(\cdot)$ be the piecewise linear path connecting $(0,0),\left(t^{*}, f\left(t^{*}\right)\right)$ and $(\bar{t}, a)$ and then dropping off with slope $-\mu$. Clearly, $h(f) \geqslant h(p)$ and $h(p) \geqslant h\left(r_{t}\right)$. We have

$$
h(p)=t^{*} I\left(\frac{f\left(t^{*}\right)}{t^{*}}\right)+\left(\bar{t}-t^{*}\right) I\left(\frac{a-f\left(t^{*}\right)}{\bar{t}-t^{*}}\right) .
$$

Because of the constraint on $f\left(t^{*}\right)$, it is easy to see that there is $\eta_{2}>0$ such that $h(p)>h\left(r_{t}\right)+\eta_{2}$. So, $h(f)>h\left(q_{a}\right)+\eta_{2}$.

Case 2: $f\left(t^{*}\right)<r_{\bar{t}}\left(t^{*}\right)-2 \epsilon / 3$ and $t^{*}<\bar{t}$.

Exactly as in case 1 , we can show that there is $\eta_{3}>0$ such that $h(f)>h\left(q_{a}\right)+$ $\eta_{3}$.

Case 3: $f\left(t^{*}\right)>r_{i}\left(t^{*}\right)+2 \epsilon / 3$ and $t^{*}>\bar{t}$.

Let $p(\cdot)$ denote the piecewise linear path connecting the points $(0,0),(\bar{t}, a)$ and $\left(t^{*}, f\left(t^{*}\right)\right)$ and then dropping off with slope $-\mu$. Clearly $h(f) \geqslant h(p)$ and $h(p) \geqslant h\left(r_{t}\right)$. We have

$$
h(p)=\bar{t} I\left(\frac{a}{\bar{t}}\right)+\left(t^{*}-\bar{t}\right) I\left(\frac{f\left(t^{*}\right)-a}{t^{*}-\bar{t}}\right) .
$$

Because of the constraint on $f\left(t^{*}\right)$, we see that there is $\eta_{4}>0$ such that $h(p)>h\left(r_{t}\right)+\eta_{4}$. So, $h(f)>h\left(q_{a}\right)+\eta_{4}$.

Case 4: $f\left(t^{*}\right)<r_{i}\left(t^{*}\right)+2 \epsilon / 3$ and $t^{*}>\bar{t}$.

Exactly as in Case 3, we can show that there is $\eta_{5}>0$ such that $h(f)>h\left(q_{a}\right)$ $+\eta_{5}$. Letting $\eta=\min \left(\eta_{1}, \eta_{2}, \ldots, \eta_{5}\right)$ completes the proof.

Finally, we have the following.

LEMMA 2.8

There is a countable set $U \subseteq R_{+}$such that, for $\epsilon \notin U, H\left(\bar{Y}_{\epsilon}^{E}\right)=H\left(Y_{\epsilon}\right)$. 


\section{Proof}

Considered as a function of $\epsilon$ for $\epsilon>0, H\left(Y_{\epsilon}\right)$ is nondecreasing. Hence there is a countable set $U \subseteq R_{+}$such that $H\left(Y_{\epsilon}\right)$ is continuous in $\epsilon$ for $\epsilon \notin U$.

Fix $0<\delta<\epsilon$. Let $g \in \bar{Y}_{\epsilon}^{E}$ and $\left\{f_{n}\right\} \subseteq \Gamma$ be such that $d_{S}\left(f_{n}, g\right) \rightarrow 0$ as $n \rightarrow \infty$. Let $\left\{g_{n}\right\} \subseteq Y_{\epsilon} \cap \Gamma$ be such that $d_{S}\left(g_{n}, g\right) \rightarrow 0$ as $n \rightarrow \infty$. Let $K=\max \left(z_{0}, \mu\right)$. Then there is $N(\epsilon, \delta)$ so large that for all $m, n \geqslant N(\epsilon, \delta)$ we have

$$
d_{S}\left(g_{m}, f_{n}\right)<\frac{\epsilon-\delta}{K} \text {. }
$$

We claim that

$$
\limsup _{n \rightarrow \infty} 1\left(f_{n} \in B\left(q_{a}, \delta\right)\right)=0
$$

i.e., $f_{n}$ eventually leaves $B\left(q_{a}, \delta\right)$. Suppose not. Then we can find $N>N(\epsilon, \delta)$ such that $f_{N} \in B\left(q_{a}, \delta\right)$. Pick $m>N(\epsilon, \delta)$ and let $t^{*}$ be such that $\mid g_{m}\left(t^{*}\right)-$ $q_{a}\left(t^{*}\right) \mid>\epsilon$. For any time changes $\lambda(\cdot)$ and $\eta(\cdot)$, and $t \in[0,1]$, we have,

$$
\begin{aligned}
\left|g_{m}(\lambda(t))-f_{N}(\eta(t))\right| \geqslant & \mid g_{m}(\lambda(t))-q_{a}\left(\lambda(t)|-| f_{N}(\eta(t))-q_{a}(\eta(t)) \mid\right. \\
& -\mid q_{a}\left(\lambda(t)-q_{a}(\eta(t)) \mid .\right.
\end{aligned}
$$

Now, $\left|q_{a}(\lambda(t))-q_{a}(\eta(t))\right| \leqslant K|\lambda(t)-\eta(t)|$. Hence

$$
\begin{aligned}
K d_{S}\left(g_{m}, f_{N}\right) & \geqslant \sup _{t \in[0,1]}\left|g_{m}(\lambda(t))-f_{N}(\eta(t))\right|+K \sup _{t \in[0,1]}|\lambda(t)-\eta(t)| \\
& \geqslant\left|g_{m}\left(t^{*}\right)-q_{a}\left(t^{*}\right)\right|-\sup _{t \in[0,1]}\left|f_{N}(t)-q_{a}(t)\right| \\
& >\epsilon-\delta .
\end{aligned}
$$

This contradiction establishes the claim.

From the claim we have $h(g) \geqslant h\left(Y_{\delta}\right)$ for every $g \in \bar{Y}_{\epsilon}^{E}$ and any $\delta<\epsilon$. If $\epsilon \notin U$, then, letting $\delta \rightarrow \epsilon$ gives $h(g) \geqslant H\left(Y_{\epsilon}\right)$ for every $g \in \bar{Y}_{\epsilon}^{E}$, so $h\left(\bar{Y}_{\epsilon}^{E}\right) \geqslant H\left(Y_{\epsilon}\right)$. It is trivial to see that $H\left(Y_{\epsilon}\right) \geqslant H\left(\bar{Y}_{\epsilon}^{E}\right)$, which completes the proof.

Proof of thm. 2.3.

Let $F: C \rightarrow R$ be a bounded continuous function, and $K<\infty$ be such that $F(g) \leqslant K$ for all $g \in C$. We have to show that $\int F \mathrm{~d} Q_{n} \rightarrow F\left(q_{a}\right)$ as $n \rightarrow \infty$. Given $\Delta>0$, we may choose $\epsilon>0, \epsilon \notin U$, such that $\left|F(g)-F\left(q_{a}\right)\right|<\Delta$ for all $g \in$ $\bar{B}\left(q_{a}, \epsilon\right)$. From lemmas 2.5 and 2.6, and thm. 2.4, we have

$$
\log P\left(s_{n}(\cdot) \in X\right)=-n h\left(q_{a}\right)+o(n)
$$

while from lemmas 2.7 and 2.8, and thm. 2.4, we have

$$
\log P\left(s_{n}(\cdot) \in Y_{\epsilon}\right) \leqslant-n\left(h\left(q_{a}\right)+\eta\right),
$$

for some $\eta>0$. Now,

$$
\begin{aligned}
\left|\int F \mathrm{~d} Q_{n}-F\left(q_{a}\right)\right| & \leqslant\left|\int_{\bar{B}\left(q_{a}, \epsilon\right)} F \mathrm{~d} Q_{n}-F\left(q_{a}\right)\right|+\left|\int_{Y_{\epsilon}} F \mathrm{~d} Q_{n}\right| \\
& \leqslant \Delta Q_{n}\left(\bar{B}\left(q_{a}, \epsilon\right)\right)+K Q_{n}\left(Y_{\epsilon}\right) \\
& =\Delta \frac{P\left(s_{n}(\cdot) \in \bar{B}\left(q_{a}, \epsilon\right)\right)}{P\left(s_{n}(\cdot) \in X\right)}+K \frac{P\left(s_{n}(\cdot) \in Y_{\epsilon}\right)}{P\left(s_{n}(\cdot) \in X\right)} .
\end{aligned}
$$


From eqns. (2.3) and (2.4), we have

$$
\underset{n \rightarrow \infty}{\limsup }\left|\int F \mathrm{~d} Q_{n}-F\left(q_{a}\right)\right| \leqslant \Delta .
$$

Letting $\Delta \rightarrow 0$ completes the proof.

\section{Regularly varying positive tail}

Throughout this section we assume the moment condition (D). Recall that a function $L(\cdot)$ is called slowly varying if

$$
\frac{L(t x)}{L(x)} \rightarrow 1 \text { as } x \rightarrow \infty \text { for any } t>0 .
$$

When $P\left(X_{1}>x\right) \sim x^{-q} L(x)$ as $x \rightarrow \infty$, with $q>0$ and $L(\cdot)$ slowly varying, $X_{1}$ is said to have regularly varying positive tail. For more about regularly varying distributions see Feller, secs. VIII.8 and VIII.9, [8].

The main theorem of this section is the following:

\section{THEOREM 3.1}

Under the moment conditions (D)

$$
\hat{P}_{n} \stackrel{w}{\rightarrow}\left[J_{T}-\mu(\cdot-T)\right] 1(T<\cdot),
$$

where $T$ is distributed on $[0,1]$ with

$$
P(T>t)=\frac{\int_{t}^{1}\left(\frac{a+s \mu}{a}\right)^{-q} \mathrm{~d} s}{\int_{0}^{1}\left(\frac{a+s \mu}{a}\right)^{-q} \mathrm{~d} s} .
$$

and $J_{T}$ is distributed on $[a+\mu(1-T), \infty)$ with

$$
P\left(J_{T}>x\right)=\left(\frac{x}{a+\mu(1-T)}\right)^{-q} .
$$

See fig. 5. Here $\stackrel{w}{\rightarrow}$ denotes weak convergence on $D$.

Heuristically, thm. 3.1 says that the reason customer $n$ incurred a waiting time of at least $n a$ is that a single (random) customer [ $k T]$ incurred an enormous waiting time. The other customers preceding customer $[k T]$ encountered typical behaviour in the queue, while the customers $k,[k T]<k \leqslant n$, encountered typical behaviour in the queue but had large waiting times because of the waiting time of customer $[k T]$. 


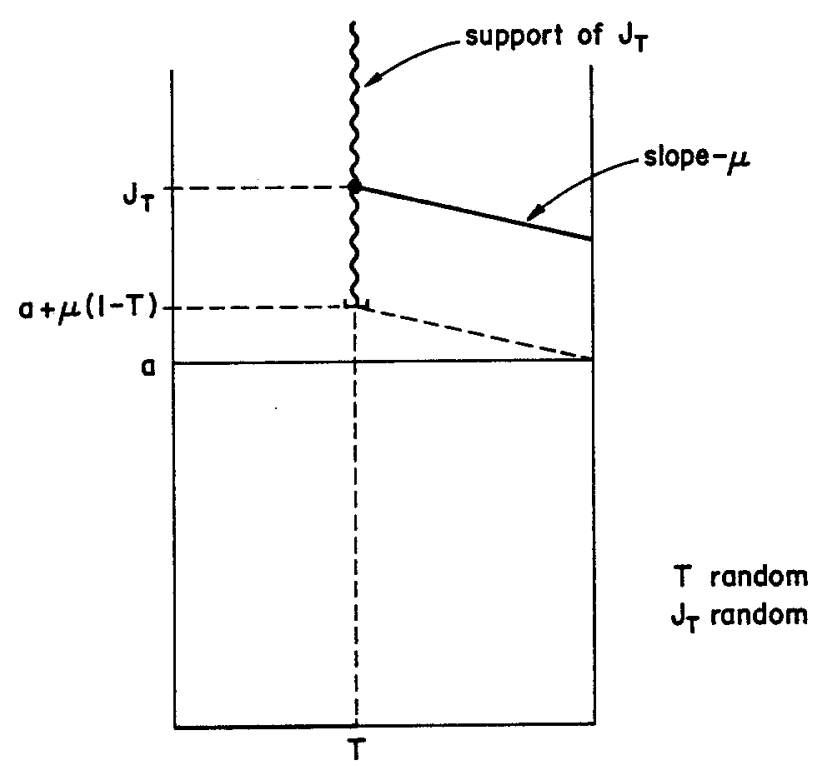

Fig. 5.

As in section 2, we can convert our problem into a problem of conditioning on large maxima in a random walk. Let $Y_{1}, Y_{2}, \ldots$ be i.i.d. with the distribution of $X_{1}$, and let:

$$
\begin{aligned}
& S_{0}=0, \\
& S_{k}=Y_{1}+Y_{2}+\ldots+Y_{k}, k \geqslant 1, \\
& M_{n}=\max _{0 \leqslant k \leqslant n} S_{k} .
\end{aligned}
$$

From the points

$$
\left(\frac{k}{n}, \frac{S_{k}}{n}\right), 0 \leqslant k \leqslant n,
$$

we construct the piecewise constant right continuous path $\hat{s}_{n}(\cdot) \in D$. Let $\hat{Q}_{n}$ denote the conditional distribution $\left(\hat{s}_{n}(\cdot) \mid M_{n}>n a\right)$. Consider the function $\hat{\Psi}: D$ $\rightarrow D$ given by

$$
\hat{\Psi}(\phi(\cdot))(t)=\left[\left[\sup _{1-t \leqslant s \leqslant 1} \phi(s)\right]-\phi(1-t)\right]^{\text {r.c. }},
$$

where $[f(\cdot)]^{\text {r.c. }}$ denotes the right continuous version of $f(\cdot)$. Then $\hat{\Psi}$ is continuous on $D$, and

$$
\hat{P}_{n}=\hat{\Psi}_{*}\left(\hat{Q}_{n}\right)
$$

The proof of this fact is identical to that of lemma 3.2.

By Billingsley, thm. 5.1, [5], thm. 3.1 reduces to the following: 
THEOREM 3.2

Under the moment conditions (D)

$$
\hat{Q}_{n} \stackrel{w}{\rightarrow} J_{1-\tau} 1(\tau<\cdot)-\mu \cdot
$$

where $\tau$ is distributed on $[0,1]$ with

$$
P(\tau<t)=\frac{\int_{0}^{t}\left(\frac{a+s \mu}{a}\right)^{-q} \mathrm{~d} s}{\int_{0}^{1}\left(\frac{a+s \mu}{a}\right)^{-q} \mathrm{~d} s} .
$$

The following theorem of Durrett, [7], will be used in the proof of thm. 3.2:

\section{THEOREM 3.3}

Under the moment conditions (D), for any $a>-\mu$,

$$
\left(\hat{s}_{n}(\cdot) \mid S_{n}>n a\right) \stackrel{w}{\rightarrow} J_{0} 1(U<\cdot)-\mu \cdot
$$

where $U$ is uniformly distributed on $[0,1]$.

Proof

This is thm. 3.1 of Durrett, [7].

To describe the essential idea in the proof of thm. 3.2, introduce the random variables

$$
\hat{Y}_{n}=\max _{1 \leqslant k \leqslant n}\left(Y_{k}-(k-1) \mu\right),
$$

and

$$
N^{a}=\#\left\{k \text { s.t. } Y_{k}>n a+(k-1) \mu\right\} .
$$

Most of the work goes into showing that

$$
P\left(M_{n}>n a\right) \sim P\left(N^{a}=1\right) \sim P\left(M_{n}>n a, N^{a}=1\right) \text { as } n \rightarrow \infty .
$$

Thus, conditioning on $\left\{M_{n}>n a\right\}$ is asymptotically equivalent to conditioning on $\left\{N^{a}=1\right\}$. This reduction yields the theorem easily because the events $\left\{Y_{k}>n a+\right.$ $(k-1) \mu\}, 1 \leqslant k \leqslant n$, are independent.

Before embarking on the proof, we need some preliminary lemmas. Lemmas 3.4 is Feller's lemma 2, pg. 277, [8].

\section{LEMMA 3.4}

The passage to the limit in eqn. (3.1) is uniform over finite intervals $t \in[a, b]$.

An easy corollary of the above is the following: 


\section{COROLLARY 3.5}

For any fixed $C$,

$$
\frac{L(t x+C)}{L(x)} \rightarrow 1 \text { as } x \rightarrow \infty \text { for any } t>0
$$

The final preliminary lemma is central to Durrett's proof of thm. 3.3, and will be used in our proof of thm. 3.2.

\section{LEMMA 3.6}

For any $a>-\mu$,

$$
P\left(S_{n}>n a\right) \sim n P\left(X_{1}>n(a+\mu)\right) \text { as } n \rightarrow \infty .
$$

Proof

Let $Y_{k}^{\prime}=Y_{k}-a, k=1,2, \ldots$, so $E Y_{k}^{\prime}=-\mu-a<0$. Also,

$$
P\left(Y_{1}^{\prime}>x\right)=P\left(Y_{1}>x+a\right) \sim(x+a)^{-q} L(x+a) \sim x^{-q} L(x) \text { as } x \rightarrow \infty,
$$

by cor. 3.5 .

Let $S_{n}^{\prime}=Y_{1}^{\prime}+\ldots+Y_{n}^{\prime}=S_{n}-n a$. Then

$$
\begin{aligned}
\frac{P\left(S_{n}>n a\right)}{n P\left(X_{1}>n(a+\mu)\right)} & =\frac{P\left(S_{n}^{\prime}>0\right)}{n P\left(Y_{1}^{\prime}>n(a+\mu)-a\right)} \\
& \sim \frac{P\left(S_{n}^{\prime}>0\right)}{n P\left(Y_{1}^{\prime}>n(a+\mu)\right)} \text { as } n \rightarrow \infty,
\end{aligned}
$$

by cor. 3.5 .

From thm. 2.1 of Durrett, [7], we see that the last quantity tends to 1 as $n \rightarrow \infty$.

We first investigate $P\left(N^{a}=1\right)$. We have:

$$
\begin{aligned}
\left\{N^{a} \geqslant 1\right\} & =\bigcup_{1 \leqslant k \leqslant n}\left\{Y_{k}>n a+(k-1) \mu\right\} \\
& =\left\{\hat{Y}_{n}>n a\right\} .
\end{aligned}
$$

\section{LEMMA 3.7}

$$
P\left(N^{a}=1\right) \sim P\left(\hat{Y}_{n}>n a\right) \text { as } n \rightarrow \infty .
$$

\section{Proof}

The result is a consequence of lemma 3.8 below. Indeed, we have

$$
P\left(N^{a}>1 \mid \hat{Y}_{n}>n a\right)=\frac{P\left(N^{a}>1\right)}{P\left(\hat{Y}_{n}>n a\right)}
$$


with

$$
P\left(N^{a}>1\right) \leqslant \sum_{1 \leqslant i<j \leqslant n} P\left(Y_{i}>n a+(i-1) \mu, Y_{j}>n a+(j-1) \mu\right)
$$

and

$$
\begin{aligned}
P\left(\hat{Y}_{n}>n a\right) \geqslant & \sum_{k=1}^{n} P\left(Y_{k}>n a+(k-1) \mu\right) \\
& -\sum_{1 \leqslant i<j \leqslant n} P\left(Y_{i}>n a+(i-1) \mu, Y_{j}>n a+(j-1) \mu\right) .
\end{aligned}
$$

From lemma 3.8, we have

$$
\frac{\sum_{1 \leqslant i<j \leqslant n} P\left(Y_{i}>n a+(i-1) \mu, Y_{j}>n a+(j-1) \mu\right)}{\sum_{k=1}^{n} P\left(Y_{k}>n a+(k-1) \mu\right)} \rightarrow 0 \text { as } n \rightarrow \infty
$$

from which the claim follows.

LEMMA 3.8

$$
P\left(\hat{Y}_{n}>n a\right) \sim \sum_{k=1}^{n} P\left(Y_{k}>n a+(k-1) \mu\right) \text { as } n \rightarrow \infty .
$$

\section{Proof}

Clearly,

$$
P\left(\hat{Y}_{n}>n a\right) \leqslant \sum_{k=1}^{n} P\left(Y_{k}>n a+(k-1) \mu\right) .
$$

Also,

$$
\begin{aligned}
P\left(\hat{Y}_{n}>n a\right) \geqslant & \sum_{k=1}^{n} P\left(Y_{k}>n a+(k-1) \mu\right) \\
& -\sum_{1 \leqslant i<j \leqslant n} P\left(Y_{i}>n a+(i-1) \mu, Y_{j}>n a+(j-1) \mu\right) \\
\geqslant & \sum_{k=1}^{n} P\left(Y_{k}>n a+(k-1) \mu\right)-\frac{n(n-1)}{2}\left[P\left(Y_{1}>n a\right)\right]^{2} .
\end{aligned}
$$

So,

$$
\begin{aligned}
\frac{P\left(\hat{Y}_{n}>n a\right)}{\sum_{k=1}^{n} P\left(Y_{k}>n a+(k-1) \mu\right)} \geqslant 1-\frac{\frac{n(n-1)}{2}\left[P\left(Y_{1}>n a\right)\right]^{2}}{\sum_{k=1}^{n} P\left(Y_{k}>n a+(k-1) \mu\right)} \\
\geqslant 1-\frac{\frac{n(n-1)}{2}\left[P\left(Y_{1}>n a\right)\right]^{2}}{n P\left(Y_{1}>n(a+\mu)\right)}
\end{aligned}
$$


Now,

$$
\begin{aligned}
\frac{P\left(Y_{1}>n a\right)}{P\left(Y_{1}>n(a+\mu)\right)} & =\left(\frac{a}{a+\mu}\right)^{-q} \frac{L(n a)}{L(n(a+\mu))} \\
& \rightarrow\left(\frac{a}{a+\mu}\right)^{-q} \text { as } n \rightarrow \infty,
\end{aligned}
$$

and

$$
n P\left(Y_{1}>n a\right) \leqslant \frac{E Y_{1}^{2}}{n a^{2}} .
$$

From (3.5), (3.6), (3.7), and (3.8), the claim follows.

We proceed to investigate $P\left(M_{n}>n a\right)$.

LEMMA 3.9

Fix $0<\epsilon<a \wedge \mu$. Then,

$$
P\left(M_{n}>n a\right) \sim P\left(M_{n}>n a, S_{n}>n(a-\mu-\epsilon)\right) \text { as } n \rightarrow \infty \text {. }
$$

\section{Proof}

Write

$$
\begin{aligned}
P\left(M_{n}>n a\right)= & P\left(M_{n}>n a, S_{n}>n(a-\mu-\epsilon)\right) \\
& +P\left(S_{n} \leqslant n(a-\mu-\epsilon) \mid M_{n}>n a\right) P\left(M_{n}>n a\right) .
\end{aligned}
$$

We first estimate $P\left(S_{n} \leqslant n(a-\mu-\epsilon) \mid M_{n}>n a\right)$. Let

$$
\tau=\inf \left\{1 \leqslant k \leqslant n: S_{k}>n a\right\},
$$

and

$$
q_{k}=P\left(\tau=k \mid M_{n}>n a\right) .
$$

Then

$$
\begin{aligned}
P & \left(S_{n} \leqslant n(a-\mu-\epsilon) \mid M_{n}>n a\right) \\
& =\sum_{k=1}^{n} P\left(S_{n} \leqslant n(a-\mu-\epsilon) \mid M_{n}>n a, \tau=k\right) q_{k} \\
& \leqslant \sum_{k=1}^{n} P\left(Y_{k+1}+\ldots+Y_{n} \leqslant-n(\mu+\epsilon)\right) q_{k} \\
& =\sum_{k=1}^{n} P\left(Y_{k+1}+\ldots+Y_{n}+(n-k) \mu \leqslant(-k \mu-n \epsilon) q_{k}\right. \\
& \leqslant \sum_{k=1}^{n} \frac{(n-k) E\left(X_{1}+\mu\right)^{2}}{(k \mu+n \epsilon)^{2}} q_{k} \\
& \leqslant \frac{E\left(X_{1}+\mu\right)^{2}}{\epsilon^{2}} \sum_{k=1}^{n} \frac{q_{k}(n-k)}{(n+k)^{2}} \\
& \leqslant \frac{1}{n \epsilon^{2}} E\left(X_{1}+\mu\right)^{2} .
\end{aligned}
$$


Thus we have,

$$
\begin{aligned}
& \frac{P\left(M_{n}>n a, S_{n}>n(a-\mu-\epsilon)\right)}{1-\frac{1}{n \epsilon^{2}} E\left(X_{1}+\mu\right)^{2}} \\
& \geqslant P\left(M_{n}>n a\right) \geqslant P\left(M_{n}>n a, S_{n}>n(a-\mu-\epsilon)\right),
\end{aligned}
$$

from which the claim follows.

From thm. 3.3 we easily get

$$
P\left(M_{n}>n a \mid S_{n}>n(a-\mu-\epsilon)\right) \rightarrow \int_{0}^{1}\left(\frac{a+s \mu}{a-\epsilon}\right)^{-q} \mathrm{~d} s,
$$

while from lemma 3.6 we have

$$
P\left(S_{n}>n(a-\mu-\epsilon)\right) \sim n P\left(Y_{1}>n(a-\epsilon)\right) \text { as } n \rightarrow \infty \text {. }
$$

Together with eqns. (3.9) and (3.10) and lemmas (3.7)-(3.9), the following lemma completes the proof of the asymptotic equivalence of $P\left(N^{a}=1\right)$ and $P\left(M_{n}>n a\right)$.

LEMMA 3.10

$$
\frac{\sum_{k=1}^{n} P\left(Y_{k}>n a+(k-1) \mu\right)}{n P\left(Y_{1}>n(a-\epsilon)\right)} \rightarrow \int_{0}^{1}\left(\frac{a+s \mu}{a-\epsilon}\right)^{-q} \mathrm{~d} s \text { as } n \rightarrow \infty .
$$

Proof

We have

$$
\frac{\sum_{k=1}^{n} P\left(Y_{k}>n a+(k-1) \mu\right)}{n P\left(Y_{1}>n(a-\epsilon)\right)}=\frac{\frac{1}{n} \sum_{k=1}^{n}\left(a+\frac{(k-1)}{n} \mu\right)^{-q} L(n a+(k-1) \mu)}{(a-\epsilon)^{-q} L(n(a-\epsilon))} .
$$

From lemma 3.4, we have

$$
\frac{\sup _{t \in[a, a+\mu]} L(n t)}{L(n(a-\epsilon))} \rightarrow 1 \text { as } n \rightarrow \infty
$$

and

$$
\frac{\inf _{t \in[a, a+\mu]} L(n t)}{L(n(a-\epsilon))} \rightarrow 1 \text { as } n \rightarrow \infty .
$$


Also,

$$
\frac{1}{n} \sum_{k=1}^{n}\left(\frac{a+\frac{(k-1)}{n} \mu}{a-\epsilon}\right)^{-q} \rightarrow \int_{0}^{1}\left(\frac{a+s \mu}{a-\epsilon}\right)^{-q} \mathrm{~d} s \text { as } n \rightarrow \infty .
$$

From (3.11), (3.12), and (3.13) the claim follows.

With the next lemma we will have established (3.4).

LEMMA 3.11

$P\left(M_{n}>n a \mid N^{a}=1\right) \rightarrow 1$ as $n \rightarrow \infty$.

Proof

Fix $b>a$. Write

$$
\begin{aligned}
& P\left(M_{n}>n a \mid N^{a}=1\right) \\
& \quad>P\left(M_{n}>n a \mid N^{a}=1, \hat{Y}_{n}>n b\right) P\left(\hat{Y}_{n}>n b \mid N^{a}=1\right) .
\end{aligned}
$$

Now,

$$
\begin{aligned}
P\left(\hat{Y}_{n}>n b \mid N^{a}=1\right) & \geqslant \inf _{1 \leqslant k \leqslant n} \frac{P\left(X_{1}>n b+(k-1) \mu\right)}{P\left(X_{1}>n a+(k-1) \mu\right)} \\
& \geqslant\left(\frac{b}{a}\right)^{-q} \inf _{1 \leqslant k \leqslant n} \frac{L(n b+(k-q) \mu)}{L(n a+(k-1) \mu)} \\
& \geqslant\left(\frac{b}{a}\right)^{-q} \inf _{x \in[n a, n(a+\mu)] \quad \inf _{t \in[b /(a+\mu),(b+\mu) / a]} \frac{L(t x)}{L(x)}} \\
& \rightarrow\left(\frac{b}{a}\right)^{-q} \text { as } n \rightarrow \infty,
\end{aligned}
$$

by lemma 3.4 .

On $\left\{N^{a}=1, \hat{Y}_{n}>n b\right\}$ there is a unique $\hat{k}, 1 \leqslant \hat{k} \leqslant n$, such that $Y_{\hat{k}}>n b+(\hat{k}-$ 1) $\mu$. Then

$$
\begin{aligned}
& P\left(M_{n}>n a \mid N^{a}=1, \hat{Y}_{n}>n b\right) \\
& =\sum_{k=1}^{n} P\left(M_{n}>n a \mid N^{a}=1, \hat{Y}_{n}>n b, \hat{k}=k\right) \\
& P\left(\hat{k}=k \mid N^{a}=1, \hat{Y}_{n}>n b\right) .
\end{aligned}
$$

Define independent random variables $\tilde{Y}_{k}, 1 \leqslant k \leqslant n$, with distribution

$$
P\left(\hat{Y}_{k} \leqslant x\right)=\frac{P\left(Y_{k} \leqslant(x-\mu) \wedge(n a+(k-1) \mu)\right)}{P\left(Y_{k} \leqslant n a+(k-1) \mu\right)},
$$

and independent random variables $Y_{k}^{*}, 1 \leqslant k \leqslant n$, with the distribution of $\tilde{Y}_{1}$. Let

$$
\tilde{S_{k}}=\tilde{Y}_{1}+\ldots+\tilde{Y}_{k},
$$


and

$$
S_{k}^{*}=Y_{1}^{*}+\ldots+Y_{k}^{*}
$$

If we let

$$
p_{k}=P\left(\tilde{S_{k}} \leqslant n(a-b)\right),
$$

then we have

$$
P\left(M_{n}>n a \mid N^{a}=1, \hat{Y}_{n}>n b, \hat{k}=k\right) \geqslant 1-p_{k-1},
$$

because the conditional distribution of $\left\{Y_{j}+\mu, 1 \leqslant j \leqslant k-1\right\}$, given $N^{a}=1$ and $\hat{k}=k$ is the distribution of $\left.\tilde{Y}_{j}, 1 \leqslant j \leqslant k-1\right\}$.

Since $E\left(Y_{1}+\mu\right)=0$, we have $E \tilde{Y}_{1}>(a-b) / 2$ for large enough $n$. So,

$$
\begin{aligned}
p_{k} & \leqslant P\left(S_{k}^{*} \leqslant n(a-b)\right)=P\left(S_{k}^{*}-k E Y_{1}^{*} \leqslant n(a-b)-k E Y_{1}^{*}\right) \\
& \leqslant P\left(S_{k}^{*}-k E Y_{1}^{*} \leqslant \frac{n(a-b)}{2}\right) \\
& \leqslant \frac{4 k \operatorname{var} Y_{1}^{*}}{n^{2}(a-b)^{2}} .
\end{aligned}
$$

Since $E Y_{1}^{2}<\infty$, there is a uniform bound on var $Y_{1}^{*}$ for all large enough $n$. Hence,

$$
\sup _{1 \leqslant k \leqslant n-1} p_{k} \rightarrow 0 \text { as } n \rightarrow \infty
$$

This, together with (3.16) and (3.17), gives

$$
P\left(M_{n}>n a \mid N^{a}=1, \hat{Y}_{n}>n b\right) \rightarrow 1 \text { as } n \rightarrow \infty .
$$

Putting (3.14), (3.15) and (3.18) together, and letting $b \rightarrow a$, yields the claim.

\section{Proof of thm. 3.2}

From (3.4), which we have established by the sequence of lemmas above, it suffices to show that

$$
\left(\hat{s}_{n}(\cdot) \mid N^{a}=1\right) \stackrel{w}{\rightarrow} J_{1-\tau} 1(\tau<\cdot)-\mu \cdot,
$$

with $\tau$ and $J_{1-\tau}$ distributed as in (3.3) and (3.2).

On $\left\{N^{a}=1\right\}$ there is a unique index $\hat{k}, 1 \leqslant \hat{k} \leqslant n$, such that $Y_{\hat{k}}>n a+(\hat{k}-1) \mu$. Clearly,

$$
\begin{aligned}
P\left(\hat{k} \leqslant j \mid N^{a}=1\right)= & \frac{\sum_{k=1}^{j} P\left(Y_{k}>n a+(k-1) \mu\right)}{\sum_{k=1}^{n} P\left(Y_{k}>n a+(k-1) \mu\right)} \\
& \frac{\frac{1}{n} \sum_{k=1}^{j}\left(a+\frac{k-1}{n} \mu\right)^{-q}}{\frac{1}{n} \sum_{k=1}^{n}\left(a+\frac{k-1}{n} \mu\right)^{-q}} \frac{\sup _{1 \leqslant k \leqslant j} L(n a+(k-1) \mu)}{\inf _{1 \leqslant k \leqslant n} L(n a+(k-1) \mu)} .
\end{aligned}
$$


Now,

$$
\frac{\sup _{1 \leqslant k \leqslant j} L(n a+(k-1) \mu)}{\inf _{1 \leqslant k \leqslant n} L(n a+(k-1) \mu)} \leqslant \sup _{x \in[n a, n(a+\mu)]} \sup _{t \in[a /(a+\mu),(a+\mu) / a]} \frac{L(t x)}{L(x)},
$$

which tends to 1 as $n \rightarrow \infty$, by lemma 3.4 .

We get a similar lower bound for $P\left(\hat{k} \leqslant j \mid N^{a}=1\right)$. Observing that

$$
\frac{\frac{1}{n} \sum_{k=1}^{[n t]}\left(a+\frac{k-1}{n} \mu\right)^{-q}}{\frac{1}{n} \sum_{k=1}^{n}\left(a+\frac{k-1}{n}, u\right)^{-q}} \rightarrow \frac{\int_{0}^{t}\left(\frac{a+s \mu}{a}\right)^{-q} \mathrm{~d} s}{\int_{0}^{1}\left(\frac{a+s \mu}{a}\right)^{-q} \mathrm{~d} s} \text { as } n \rightarrow \infty,
$$

we have

$$
P\left(\hat{k} \leqslant[n t] \mid N^{a}=1\right) \rightarrow \frac{\int_{0}^{t}\left(\frac{a+s \mu}{a}\right)^{-q} \mathrm{~d} s}{\int_{0}^{1}\left(\frac{a+s \mu}{a}\right)^{-q} \mathrm{~d} s} .
$$

The big jump $Y_{\hat{k}}$ satisfies

$$
\begin{aligned}
P\left(Y_{\hat{k}}>n b \mid N^{a}=1, \hat{k}=k\right) & =\frac{P\left(Y_{1}>n b\right)}{P\left(Y_{1}>n a+(k-1) \mu\right)} \\
& =\left(\frac{b}{a+\frac{k-1}{n} \mu}\right)^{-q} \frac{L(n b)}{L(n a+(k-1) \mu)} .
\end{aligned}
$$

From (3.19) and (3.20) it follows that

$$
\left(\frac{Y_{\hat{k}}}{n} 1(\hat{k} \leqslant n \cdot)-\mu \cdot \mid N^{a}=1\right) \stackrel{w}{\rightarrow} J_{1-\tau} 1(\tau<\cdot)-\mu \cdot,
$$

with $\tau$ and $J_{1-\tau}$ distributed as in (3.3) and (3.2). To complete the proof of the theorem it suffices to establish that

$$
\left(\hat{s}_{n}(\cdot)-\left[\frac{Y_{\hat{k}}}{n} 1(\hat{k} \leqslant n \cdot)-\mu \cdot\right] \mid N^{a}=1\right) \stackrel{w}{\rightarrow} \delta_{0},
$$

where $\delta_{0}$ denotes the probability distribution concentrated on the zero function. This is done in lemma 3.12 below.

\section{LEMMA 3.12}

Let $\hat{k}, 1 \leqslant \hat{k} \leqslant n$, denote the unique index on $\left\{N^{a}=1\right\}$ such that $Y_{\hat{k}}>n a+(\hat{k}$ -1) $\mu$. Then,

$$
\left(\hat{s}_{n}(\cdot)-\left[\frac{Y_{\hat{k}}}{n} 1(\hat{k} \leqslant n \cdot)-\mu \cdot\right] \mid N^{a}=1\right) \stackrel{w}{\rightarrow} \delta_{0}
$$




\section{Proof}

Let $\tilde{Y}_{k}, 1 \leqslant k \leqslant n$, be independent random variables with distribution

$$
P\left(\tilde{Y}_{k} \leqslant x\right)=\frac{P\left(Y_{k} \leqslant(x-\mu) \wedge(n a+(k-1) \mu)\right)}{P\left(Y_{k} \leqslant n a+(k-1) \mu\right)} .
$$

Let

$$
\begin{aligned}
& \tilde{S_{0}}=0, \\
& \tilde{S}_{k}=\tilde{Y}_{1}+\cdots+\tilde{Y}_{k},
\end{aligned}
$$

and consider the piecewise constant, right continuous path $\tilde{s}_{n}(\cdot)$ constructed from the points $\left(k / n, \tilde{S}_{k} / n\right), 0 \leqslant k \leqslant n$. Let $\tilde{k}$ be independent of $\left(\tilde{Y}_{1}, \ldots, \tilde{Y}_{n}\right)$, taking values in $\{1, \ldots, n\}$, with distribution

$$
P(\tilde{k}=k)=\frac{P\left(X_{1}>n a+(k-1) \mu\right)}{\sum_{j=1}^{n} P\left(X_{1}>n a+(j-1) \mu\right)} .
$$

We erase the jump $\tilde{Y}_{\tilde{k}}$ and consider the resulting path

$$
\tilde{s}_{n}(\cdot)-\frac{\tilde{Y}_{k}}{n} 1([n \cdot] \geqslant \tilde{k}) \text {. }
$$

The distribution of this path is clearly the same as the distribution

$$
\left(\hat{s}_{n}(\cdot)-\left[\frac{Y_{\hat{k}}}{n} 1(\hat{k} \leqslant n \cdot)-\mu \frac{[n \cdot]}{n}\right] \mid N^{a}=1\right) .
$$

Hence, to prove the lemma, it suffices to show that, for any $\epsilon>0$,

$$
P\left(\sup _{0 \leqslant t \leqslant 1}\left|\tilde{s}_{n}(t)-\frac{\tilde{Y}_{\hat{k}}}{n} 1([n t] \geqslant \tilde{k})\right|>\epsilon\right) \rightarrow 0 \text { as } n \rightarrow \infty,
$$

or, equivalently, that

$$
P\left(\sup _{0 \leqslant k \leqslant n}\left|\tilde{S}_{k}-\tilde{Y}_{\tilde{k}} 1(k \geqslant \tilde{k})\right|>n \epsilon\right) \rightarrow 0 \text { as } n \rightarrow \infty .
$$

In our notation we suppressed the dependence of the distribution of $\tilde{Y}_{k}$, $1 \leqslant k \leqslant n$, on $n$. From $E X_{1}^{2}<\infty$, it is easy to see that there is $K<\infty$ such that $E\left|\tilde{Y}_{k}\right|^{2}<K$ for all $1 \leqslant k \leqslant n$ and all $n$. By Chebyshev's inequality, see Feller, pg. $151,[8]$, we have,

$$
\frac{n^{2} \epsilon^{2}}{4} P\left(\left|\tilde{Y}_{k}\right|>\frac{n \epsilon}{2}\right) \leqslant K \text { for all } 1 \leqslant k \leqslant n \text { and all } n .
$$

So,

$$
\sup _{1 \leqslant k \leqslant n} P\left(\left|\tilde{Y}_{k}\right|>\frac{n \epsilon}{2}\right) \rightarrow 0 \text { as } n \rightarrow \infty
$$


Next, note that $\tilde{S}_{k}^{2}, 0 \leqslant k \leqslant n$, is a positive integrable supermartingale. By Doob's inequality, see Neveu, lemma IV-2-9, [10],

$$
\frac{n^{2} \epsilon^{2}}{4} P\left(\sup _{1 \leqslant k \leqslant n}\left|\tilde{S_{k}}\right|>\frac{n \epsilon}{2}\right) \leqslant E \tilde{S}_{n}^{2} \leqslant n K .
$$

So,

$$
P\left(\sup _{1 \leqslant k \leqslant n}\left|\tilde{S_{k}}\right|>\frac{n \epsilon}{2}\right) \rightarrow 0 \text { as } n \rightarrow \infty .
$$

The lemma follows directly from eqns. (3.21) and (3.22).

\section{Concluding remarks}

Soren Asmussen has kindly pointed out that theorem 2.3 can also be directly derived by an application of Wald's identity for the exponentially transformed random walk and a central limit theorem for the time of level crossing, [1]. The techniques for such a derivation are available in [2] and [3], see especially [2], cor. 3.1. Using these techniques, it is also possible to get somewhat more information, for example when $a \leqslant z_{0}$ one gets a Brownian bridge as correction to the straight line.

Proofs of theorem 2.3 under the assumption that the moment generating function $m(t)$ is defined for all $t$ are available in the papers of Borovkov, [7], and Picard and Deshayes, [11]. It appears that the technique of Asmussen, [1], will yield the result under the assumption that $m(t)$ is defined for an interval of type $\left[0, t_{+}\right)$, with $t_{+}>0$ and $m^{\prime}(t) / m(t) \rightarrow \infty$ as $t \rightarrow t_{+}$, which is weaker than our condition $(\mathrm{C})$.

\section{References}

[1] S. Asmussen, Private Communication, 1988.

[2] S. Asmussen, Conditioned limit theorems relating a random walk to its associate, with applications to risk reserve processes and the GI/G/1 queue, Advances in Applied Probability 14 (1982) 143-170.

[3] S. Asmussen, Applied Probability and Queues (John Wiley and Sons, 1987).

[4] H. Bergstrom, Weak Convergence of Measures (Academic Press, 1982).

[5] P. Billingsley, Convergence of Probability Measures (John Wiley and Sons, 1968).

[6] A.A. Borovkov, Boundary value problems for random walks and large deviations in function spaces, Theory of Probability and its Applications 12, No. 4 (1967) 575-595.

[7] R. Durett, Conditional limit theorems for random walks with negative drift, Zeit. fur Wahr. und Ver. Geb. 52 (1980) 277-287.

[8] W. Feller, An Introduction to Probability Theory and its Applications, Vol. II (John Wiley and Sons, 1971). 
[9] A.A. Mogulskii, Large deviations for trajectories of multidimensional random walks, Theory of Probability and its Applications XXI, No. 2 (1976) 300-315.

[10] J. Neveu, Discrete Parameter Martingales (North-Holland, 1975).

[11] D. Picard and J. Deshayes, Grandes et moyennes deviations pour les marches aleatories, in: Grandes Deviations et applications statistiques (Seminaire Orsay 1977-1978, Asterisque, Vol. 68, 1979) pp. 53-71. 